\title{
Role of Tobacco in the Development of Oral Leukoplakia and Oral Cancer
}

\section{Maka Sabashvili ${ }^{*}$, Elene Gigineishvili ${ }^{2}$, Maia Jikia ${ }^{3}$ and Tamta Chitaladze ${ }^{4}$}

${ }^{1}$ School of Health Sciences and Public Health, Administration of Rotational Learning, University of Georgia, DDM, Georgia

${ }^{2}$ Department of Dentistry, Administration of Rotational Learning, University of Georgia, DDM, Georgia

${ }^{3}$ Scientific Research Division, Administration of Rotational Learning, University of Georgia, DDM, Georgia

${ }^{4}$ Dental Simulation Laboratory, Administration of Rotational Learning, University of Georgia, DDM, Georgia

\begin{abstract}
Tobacco smoking is associated with the development of many diseases, including oral cancer and leukoplakia. This fact is described in the 2014 annual report of the US Office of the Surgeon General "The results of smoking on health, 50 years of progress". Despite the fact that different forms of tobacco(cigarettes, cigars, pipes, dipping and chewing tobacco or inhaled) are used in order to reduce pulmonary and cardiac complications, tobacco has a negative influence on human health. Tobacco smoking increases the death rate of smokers by $30-80 \%$. Based on the mentioned we can determine the causal relationship between smoking and the development of oral cancer, leukoplakia and chronic impact on oral organs, i.e. lips during constant tobacco use.
\end{abstract}

Keywords: Tobacco; Tobacco use; Oral leukoplakia; Oral cancer

\section{Introduction}

Smoking is associated with the development of many diseases such as cancer, cardiopulmonary diseases, etc., which lead to high mortality rate [1]. Tobacco consumption is especially high in countries with low levels of education. In developing countries high rates of tobacco use is detected among men. Tobacco industry is growing at a faster pace and the number of smokers among women and adolescents is increasing [2]. Any form of tobacco (chewing, smoking, smelling) affects oral cavity and causes life-threatening diseases such as oral cancer and leukoplakia [3]. The most important measure for preventing the oral health pathology caused by tobacco consumption is the termination of use of tobacco products. The risk of oral cancer is rapidly decreasing when smoker stops tobacco use [4]. Ex-smokers' and non-smokers' oral cavity health is the same after 10 years of smoking cessation. In this case, the risk of developing oral leukoplakia and oral cancer is minimized. It is not easy to stop tobacco use. Its cessation will result in immediate consequences, including increased life expectancy and prevention of tobacco-related diseases [1]. Harmful effects of tobacco are growing annually, but no matter how paradoxical this may sound, its consumption also increases. The age of smokers gets lower every year and the frequency of tobacco use is increased in 13-15 age group [5]. Cardiovascular diseases, hypertension, liver cirrhosis, lung cancerthis is an incomplete list of serious diseases that are often developed among chronic tobacco users [6].

The purpose of this article is to study and review the evidence of the effects of active and passive tobacco consumption on the pathology of the oral cavity, namely the development of cancer and leukoplakia. According to the statistical data, the risk of oral cancer is about twenty times higher among tobacco smokers, compared to non-smokers. In oral cavity the risk of developing gum disease, teeth caries and erosive damage is increased. Oral cancer is one of the frequent type of diseases. Among every four patients three are smokers. In case of smoking of more than one pack cigarette per day, the risk of developing oral cancer increases 30 times [7].

\section{Goal}

Why did we choose these two disease from many nosologies? These two diseases were chosen for several specific reasons, namely:

- Oral cancer and leukoplakia are related to each other. Leukoplakia is a pre-cancerous disease. Once it has been diagnosed, continuing tobacco smoking increases the risk of cancer development. Oral leukoplakia (OL) is the most well-known potentially malignant condition, which is transformed into oral cavity cancer [8]. The probability of transformation from leukoplakia into an oral cavity cancer is $0.3 \%$ to $25 \%$ [9].

- As mentioned above, leukoplakia is pre-cancerous condition. So smoking cessation contributes to the regression of Leukoplakia (up to $60 \%)$ and reduces the risk of cancer development $[10,11]$. Leukoplakia decreases gradually and completely disappears within 8-12 months of smoking cessation [12].

- Tobacco use is the main reason for development of both diseases. Smoking and alcohol consumption increases the risk of cancer in $80 \%$ of the population. This has been confirmed by the International Agency for Research on Cancer [13].

Side effects of smoking are serious. It leads to the development of disease and disability and harms nearly every organ of the body. Smoking causes cancer, heart disease, stroke, lung diseases, diabetes, and chronic obstructive pulmonary disease (COPD), which includes emphysema and chronic bronchitis. Smoking also increases risk for tuberculosis, certain eye diseases (Graves' ophthalmopathy, Dry Eye Syndrome), and diseases of the immune system, including rheumatoid arthritis. Smoking doubles the risk of developing rheumatoid arthritis [14]. Cancer can be developed in the following organs: nose, mouth, throat, trachea, esophageal, bronchials, liver, stomach, pancreas, kidneys, urinary bladder, throat, bone marrow and blood, intestines. Tobacco also affects the autoimmune system, cardiovascular system, reproductive system, respiratory system. Infectious diseases are frequently developed in smokers. Smoking also causes several autoimmune diseases, including Crohn's disease.

Many studies have shown a relation between smoking and mental illness. Half of them reported that depression/anxiety is closely linked

${ }^{*}$ Corresponding author: Maka Sabashvili, Administration of Rotational Learning University of Georgia, Dentistry, kostava str. 77, Tbilisi, Georgia, Tel: 995593224644; E-mail: m.sabashvili@ug.edu.ge

Received May 30, 2018; Accepted June 15, 2018; Published June 20, 2018

Citation: Sabashvili M, Gigineishvili E, Jikia M, Chitaladze T (2018) Role of Tobacco in the Development of Oral Leukoplakia and Oral Cancer. Dentistry 8: 495. doi:10.4172/2161-1122.1000495

Copyright: (c) 2018 Sabashvili M, et al. This is an open-access article distributed under the terms of the Creative Commons Attribution License, which permits unrestricted use, distribution, and reproduction in any medium, provided the original author and source are credited. 
to smoking [15]. The relation between smoking and mental illness is one of the main concerns of public health. Smoking is associated with a reduction in life expectancy and development of mental illness. It is reported that persons with mental illness begin smoking and consuming alcohol from early age [16].

Most recent studies show direct relation between tobacco use and decrease in bone density. Smoking is one of the many factors that increase the risk of osteoporosis. Smoker men and women appeared to have a decrease in bone mass. Cessation of smoking reduces the risk of bone clearing and fracture. Smoker women suffer from osteoporosis at an early age. Smoking also causes decrease in the level of estrogen and menopause occurs at an early age [17]. Tobacco smoke damages the blood cells with consequent deterioration of heart function, increasing the risk of formation of atherosclerotic plaques, aneurysm, cardiovascular diseases and stroke [14].

\section{Epidemiologic Patterns of Tobacco Habits on Oral Health}

In the 16th century in United States tobacco was discovered by Spanish explorer Hernandez de Bonkalo. Initially people smoked tobacco with a pipe, after they chewed and smelled it. As a form of cigarette, tobacco first appeared in Spain in the 17th century and smoking immediately became very popular habit. The relation between tobacco use and mouth cancer was first reported in the early 18 th century when smokers had been diagnosed with lip cancer. There were also many other illnesses that triggered tobacco [18].

Smoking causes many diseases and pathologies of the oral cavity. Tobacco is a risk factor for cancer of oral cavity, periodontal diseases and congenital abnormalities among children whose mothers consumed tobacco during pregnancy. It also causes periimplantitis, erythroplakia, hyperkeratosis, leukoplakia, loss of teeth and xerostomy. The frequency of development of tobacco-related diseases is increasing, especially in developing countries where high rate of tobacco consumption is observed. It is likely that in the third decade of this century tobacco is the leading cause of death that stands as a reason of higher mortality rate and high incidence of HIV, malaria, tuberculosis, maternal mortality, traffic accidents, homicide and suicide cases compared to the previous periods [19]. The effects of tobacco consumption on the oral cavity of the population are alarming. Tobacco consumption leads to gingivitis, parodontitis and parodontosis, which lead to bone depletion and loss of teeth. It begins with breaking the bone and bone tissue of the "pillar apparatus"-atrophy, the teeth begin losing and fall out of the bolt. In addition, it can adversely affect the clinical results of non-surgical and surgical treatment, as well as the implant duration [20]. Due to the variety of cancer, many of the risk factors for its development, such as ultraviolet rays or sunburns, can cause skin cancer. The risk factors include age and race. Impact of these factors is more or less different from each other [21]. Despite of these terrifying results, the number of cigarettes sales annually exceeds 5.8 trillion worldwide [22].

\section{Epidemiologic Patterns of Tobacco Habits on Leukoplakia}

As a result of tobacco consumption, a high rate of mortality caused by oral cavity was observed [6]. Therefore, tobacco is identified as a major risk factor for other diseases and possible precipitating factors, such as oral cavity leukoplakia. Leukoplakia often precedes cancer of the oral cavity, and has a similar cause [5]. Leukoplakia is a predominantly white lesion of the oral mucosa that cannot be characterized as any other definable lesion. That is a response to chronic irritant factor.
Comparing to other pre-cancerous lip diseases, leukoplakia is more commonly spread and is estimated to reach about $13 \%$ of all the oral mucosa and lip diseases. Most possibly, Leukoplakia mainly affects men over 40 years of age [23]. Leukoplakia is the most common, potentially malignant oral lesions. High rates of transformation can be seen especially when there are red areas, and it has a very low potential to be transformedinto malignant when there are white areas $[9,24]$. There are four clinical forms of leukoplakia: 1 . flat, white patch/plaque without red components; 2 . Flat, white patch/plaque with erosion or red components; 3 . Slightly raised or elevated white patch/plaque; 4. Markedly raised or elevated white patch/plaque. Different from is smokers' leukoplakia, ("Tappeiners leukoplakia"). It develops over a hard palate mucosa. This form of leukoplakia occures in individuals who smoke large amount of tobacco, especially with a pipe. According to Borovski, "Tappeiners leukoplakia" rapidly disappears after cessation of smoking [25,26].

In a study conducted in 1996, 5034 individuals were interviewed, from which 167 were found to be between the smokers and the nonsmokers 5 [7].

Leukoplakia treatment is most successful when a lesion is found and treated early, when it's small in size. Regular checkups are important, the routine inspection of mouth for areas that don't look normal.

For most people, removing the source of irritation $\nabla$ such as stopping tobacco or alcohol use improves the pathologic condition [27]. Properly planned and actively produced complex treatments have resulted positively in $88 \%$ of patients. A remarkable improvement was observed in $43 \%$ of patients and the recovery in $45 \%$. Leukoplakia disappears gradually, with the termination of irritating impact in 8-12 months [28].

\section{Epidemiologic Patterns of Tobacco Habits on Oral Cancer}

Tobacco use is one of the biggest challenges of public health. According to the most recent estimation conducted by the World Health Organization (WHO), 4.9 million people worldwide died in 2000 as a result of addiction to nicotine (WHO, World Health Report, T 2002). By 2030 this number will grow up to 10 million [28]. As a result of the active nicotine consumption, by both genders, the number of incidence of oropharyngeal cancer is the sixth in the world, while in developing countries it is the most common among men and is in the third place. In industrialized countries, tobacco consumption is predominant among men compared to women [29]. In 2004, the risk of developing oral cancer among the population of European countries was: $1.85 \%$ men and $0.37 \%$ women [30]. Tobacco and alcohol use are two main risk factors for oral cavity and oropharyngeal cancers [31]. Signs and symptoms that may indicate cancer of the oral cavity, according to the American Dental Association (ADA): A sore or tenderness in the mouth that doesn't get better; Pain, tenderness, or numbness in the mouth or on the lips; A lump or leathery patch inside mouth, or color changes in oral tissues (gray, red, or white patches, rather than a healthy pink color), having difficulty in chewing, swallowing, or speaking. If a person has any of these signs or symptoms, it's necessary to visit your health care provider or dentist [32]. Persons with oral cancer often report that the disease is preceded by mucosal trauma. The main causes of such trauma are caries teeth, incorrectly covered bone, improper prostheses, and various harmful habits, such as tobacco smoke. The above factors usually damage the mucous membranes and often cause considerable pre-cancerous processes. 
About 275,000 oral cavity damageis diagnosed every year in the world. In Sri Lanka, India, Pakistan and Bangladesh nearly a quarter of total diagnosis of cancer is the oral cavity cancer [4]. Oral cancer incidence has been examined in 2004-2008 and was found out that more than half of them were diagnosed in males of age 45-64. Some cancer cells have the ability to migrate, which can move through blood circulation and lymphatic system from one to other parts of the body. This type of cancer is distinguished as a more severe form with high mortality rate [33]. Oral mucosa cancer group includes malignant tumors that develop in the mucous membrane of the cheeks, floor of the mouth, upper and lower jaws, alveolar edges, hard and soft palate, the palate of the front rings. The most common is malignant histological type of squamous cornified malignant cancer. Currently used methods for the treatment of oral cavity cancer is the mixed method (radiation and surgery). Results of the treatment depend on the tumor histological structure, its location, form of growth presence or absence of metastases [34]. Harold summarized the results of treatment of 804 patients and found out that patients with 5 -year viability made up 35\%. In cases where regional metastasis wasn't observed, $50 \%$ of the patients lived for 5 years. Only $9.5 \%$ of patients with metastases to regional lymph nodes lived for 5 years [35]. Tobacco users die prematurely and deprive their families' income, raise the cost of health care and hinder economic development.

\section{Discussion}

\section{Tobacco habits: Statistical data}

According to the World Health Organization, Georgia occupies one of the leading positions among the smoker countries $57.7 \%$ [18]. According to The World Health Organization and various basic research data, today about $37-40 \%$ of Georgian population is smoking tobacco. Men smokers make about 50-65\% of all male population, while women smokers make about 16-22\%. The difference between men and women gradually has reduced, because the number of smoking women is increasing on a daily basis [36].

According to statistical data, the critical situation is among adolescents, especially among girls, where the rate of smokers was increased for $9 \%$ during last five years. The number of adolescent smokers reached $42.4 \%$ [37]. The epidemic of tobacco use is one of the greatest threats to global health. The World Health Organization has increased its effective control of tobacco use.

In 2000, 4.9 million people died as a result of nicotine consumption worldwide. High mortality rate is increasing rapidly, especially in underdeveloped countries [38]. In the United States the number of smokers has considerably decreased and reached $8.1 \%$ from 2000 to 2015 [15]. Indonesia is on the first place-76.2\%, while the last place takes Ethiopia-8.9\% [19].

In the United Kingdom only $0.5 \%$ of 11 -year-olds smoke regularly. By the age of 15 , this number has risen to $10 \%$, with girls more likely to smoke than boys [39-41]. As a result of tobacco consumption, 8.4 million people will die from tobacco-related illnesses by 2020. Despite intensive warnings and health risks description caused by tobacco consumption, 600 billion cigarettes are sold daily in the United States. About 23\% of American youth regularly use tobacco [36].

\section{Prevalence of oral cancer}

According to one survey, oral leukoplakia has occurred in men over 40 years of age and in women over 50 years of age. Tobacco smoking is the most described etiological factor in development of oral leukoplakia.
Smokers have a six-fold increase of the risk of developing leukoplakia of the oral mucosa compared to non-smokers. Six European studies, found a prevalence of smoking between 56-97\% in leukoplakia patients. It also showed that the majority of smokers with leukoplakia (74.0\%) smoked more than 20 cigarettes per day compared to $34.5 \%$ of those without leukoplakia [42-44]. A total of 315 cases of Oral Leukoplakia were retrieved from the Oral Pathology Service at the University of São Paulo.

A total of 131 cases involved dysplastic lesions (74 smokers and 57 non-smokers), and 184 cases described non-dysplastic lesions (96 smokers and 88 non-smokers). The frequencies of lesions, according to the grade of dysplasia, were similar in both groups [45].

Over a 30-year period, increasing prevalence of leukoplakia among the 50-60 year-old group has been identified, but the risk of malignant transformation was highest among the 60-70 age group [46].

Studies conducted in India have found that approximately 2-12\% of leukoplakia cases are malignant during several years. The prevalence of leukoplakia is described in many studies. For example, in one study, which was conducted in India, it was revealed that over one year period from 100000 men leukoplakia has been diagnosed 240 times, and from 100000 women only three times. Second study was conducted in Japan, where the diagnosis of leukoplakia was estimated at 409 out of 100000 examples and in women it was from 100000 to 70 . Worldwide, the prevalence of leukoplakia varies from 1 to $5 \%[47,48]$.

In USA the prevalence of leukoplakia among 35 years-old white women was fixed in $2.9 \%$, and the intensity of its spread in black people is not studied. In white patients $82 \%$ of leukoplakia damages are related to tobacco smoking and in black patients only $62 \%$ of leukoplakia lesions are associated with tobacco smoking. Another study, in which 197 smokers were examined, revealed that only 31 patients were diagnosed with leukoplakia.

\section{Prevalence of oral leukoplakia}

In the United States 2-4\% of cancer diagnosis of all types of cancer is due to oral cancer. Oral cancer is one of the most common types characterized with high mortality rate. The average life expectancy of patients is 5 years. Such a result is not due to the complexity of the treatment, but to the fact that the diagnoses were performed on the late stage. Similarly to many other mouth diseases, tobacco use is also the most important risk factor [12]. Smoking causes disease and disability, and damages almost all internal organs of the body. More than 16 million Americans live with the disease, caused by smoking. Cigarettes cause cancer, heart disease, stroke, diabetes and chronic obstructive pulmonary disease (COPD), which includes emphysema and chronic bronchitis. Smoking also causes tuberculosis, eye diseases, and immune system depression [33].

Secondary tobacco smoke contributes to about 41,000 adolescent and 400 infant deaths [33]. The Government of The United States spends about 170 billion dollars annually on medical services on treatment of adults' smoking-related diseases. The latest data from the American Cancer Society on oral cancer for 2018 is as follows: approximately 51.540 people developed oral cancer, and 10.030 died. This type of cancer is two times higher among men compared to women, and their number is similar among blacks and whites. The average age of people diagnosed makes 62 years old, but the disease can occur in young people as well.

Percentage of cancervary between countries. For example, it is 
more common in Hungary and France than in the United States, much less in Mexico and Japan [20,21].

In recent decades, some countries have recorded the increased incidence of cancer of the oral cavity, about $50 \%$ of patientsdiagnosed die each year. In Brazil 14,170 cases of oral cancer were reported in 2012, and in the north region there were 2550 new cases. Among them 1640 were males and 910 female. Approximately $80 \%$ of oral cavity is transformed into malignant form.

In Spain the number of deaths from oral cavity has increased dramatically from $46 \%$ to $71 \%$ over the last 10 years[22]. In 11 countries from South-East Asia exist 5 National Cancer Registry, where the cases of oral cancer were reported from 2002 to 2014. In this period 300373 new cases of oral cavity cancer and 145353 cases of mortality were fixed $[23,24]$. One study examined 310 smokers diagnosed with cancer of the oral cavity 176 .

\section{Conclusions and Recommendations}

Periodontal diseases, dental implant failure, oral cancers, precancerous diseases and leukoplakia are closely related to tobacco consumption. Advising patients to quit tobacco use is a dental professional responsibility and the dentists may play active role in nicotine replacement counselling. Smoking cessation should be incorporated as an integral teaching component of the undergraduate dental curriculum, particularly with respect to the prevention and diagnosis of tobacco-induced oral lesions and complications. Close collaboration of both dentists and general physicians with smoking cessation programs is advocated in the treatment of tobacco-smoking patients. According to one survey, $30.03 \%$ of the examined population had oral mucosal lesions with a clear association to harmful habits (tobacco and alcohol), inadequate oral hygiene practices and exposure to the sun. Initiatives directed towards prevention of disease and promotion of health should be undertaken.

In order to protect oral cavity, we need to follow three main principles:

1) to stop smoking- the risk of oral health problems decreases significantly after smoking cessation;

2) tovisit dentist regularly, as the early detection of oral cavity diseases will promote successfully management of disease;

3) to clean teeth twice more often in order to avoid accumulation of chemical residues in the mouth.

It is important to discuss these recommendations actively in public and in educational institutions to provide disease prevention and ultimate treatment. Significant restrictions should be posed to interfere with tobacco sales and the increase in tobacco taxation policy is also essential [36].

\section{References}

1. Rahmanian SD, Diaz PT, Wewers M E (2011) Tobacco use and cessation among women: research and treatment-related issues. J Womens Health 20 : 349-357.

2. Al-Sheyab N, Alomari MA, Shah S, Gallagher P, Gallagher R (2014) Prevalence, Patterns and Correlates of Cigarette Smoking in Male Adolescents in Northern Jordan, and the Influence of Waterpipe Use and Asthma Diagnosis: A Descriptive Cross-Sectional Study. Int J Environ Res Public Health 11: 90089023.

3. Awan K (2011) Effects of tobacco use on oral health - an overview. Research Gate 18: 18-23.
4. World Health Organization. Fact sheet about health benefits of smoking cessation.

5. Martin TA, Ye L, Sanders AJ, Lane J, Jiang WG (2013) Cancer Invasion and Metastasis: Molecular and Cellular Perspective. US National Library of Medicine National Institutes of Health.

6. Centers for Disease Control and Prevention. Smoking and Tobacco Use. Youth and Tobacco Use.

7. Centers for disease control and Prevention. Health Effects of Cigarette Smoking.

8. Centers for Disease Control and Prevention. Smoking and Tobacco Use Health Effects of Cigarette Smoking.

9. The Statistics Portal. Tobacco Industry - Statistics \& Facts.

10. Brouns EREA (2013) Oral Leukoplakia: a clinical, histopathological and molecular study. VU University Medical Centre and Academic Centre for Dentistry Amsterdam (ACTA), Amsterdam, the Netherlands.

11. Bokor-Bratic M, Vuckovich N (2002) Cigarette smoking as a risk factor associated with oral leukoplakia. Archive of Oncology 10: 67-70.

12. Harris KK, Zopey M, Friedman TC (2016) Metabolic effects of smoking cessation. Nat Rev Endocrinol 12: 299-308.

13. Blot WJ, McLaughlin JK, Austin DF, Greenberg RS, Preston-Martin S, et al. (1988) Smoking and drinking in relation to oral and pharyngeal cancer. Cancer Res 48: 3282-3287.

14. Di Giuseppe D, Discacciati A, Orsini N, Wolk A (2014) Cigarette smoking and risk of rheumatoid arthritis: a dose-response meta-analysis. Arthritis Res Ther 16: R61.

15. Ziedonis D, Hitsman B, Beckham JC, Zvolensky M, Adler LE, et al. (2008) Tobacco use and cessation in psychiatric disorders: National Institute of Mental Health report. Nicotine Tob Res 10: 1691-1715.

16. Mangerud WL, Bjerkeset O, Holmen TL, Lydersen S, Indredavik MS (2014) Smoking, alcohol consumption, and drug use among adolescents with psychiatric disorders compared with a population based sample. J Adolesc 7: 1189-1199.

17. Windham GC, Mitchell P, Anderson M, Lasley BL (2005) Cigarette Smoking and Effects on Hormone Function in Premenopausal Women. Environ Health Perspect 113: 1285-1290.

18. Mortazavi H, Baharvand M, Mehdipour M (2014) Oral Potentially Malignant Disorders: An Overview of More than 20 Entities. J Dent Res Dent Clin Dent Prospects 8: 6-14.

19. Proctor RN (2013) The history of the discovery of the cigarette-lung cancer link: evidentiary traditions, corporate denial, global toll. BMJ Journal 21: 87-91.

20. Malhotra R, Kapoor A, Grover V, Kaushal S (2010) Nicotine and periodontal tissues. J Indian SocPeriodontol 14: 72-79.

21. Patton GC, Coffey C, Sawyer SM, Viner RM, Haller DM, et al. (2009) Global patterns of mortality in young people: a systematic analysis of population health data. Lancet 374: 881-892.

22. Bray F, Ren JS, Masuyer E, Ferlay J (2013) Global estimates of cancer prevalence for 27 sites in the adult population in 2008. Int $\mathrm{J}$ Cancer 132: 11331145.

23. Lima JS, Pinto Ddos S Jr, Sousa SO, Corrêa L (2012) Oral leukoplakia manifests differently in smokers and non-smokers. Braz Oral Res 26: 543-549.

24. Waldron CA, Shafer WG (1975)A clinicopathologic study 3256 oral leukoplakias. Cancer 36: 1386-1392.

25. Wright JM, Dermatol S, Sciubba JJ (1994) Oral precancerous lesions and conditions. Semin Dermatol 13: 125-131.

26. Amagasa T, Yamashiro M, Shikawa H (2006) Oral Leukoplakia Related to Malignant Transformation. Oral Science International 3: 45-55.

27. Borovski E, Mashkileison A (2003) Leukoplakia. Oral cavity mucous membranes and lip diseases, pp. 234-241.

28. Bánóczy J, Gintner Z, Dombi C (2001) Tobacco Use and Oral Leukoplakia. J Dent Educ 65: 322-327. 
Citation: Sabashvili M, Gigineishvili E, Jikia M, Chitaladze T (2018) Role of Tobacco in the Development of Oral Leukoplakia and Oral Cancer. Dentistry 8: 495. doi:10.4172/2161-1122.1000495

29. Garavello W, Bertuccio P, Levi F, Lucchini F, Bosetti C, et al. (2009) The Oral cancer epidemic in central and eastern Europe. Int J Cancer 127: 160-171.

30. Sham AS, Cheung LK, Jin LJ, Corbet EF (2003) The effects of tobacco use on oral health. Hong Kong Med J 9: 271-277.

31. Krishna RSV, Mejia G, Roberts-Thomson K, Logan R (2013) Epidemiology of Oral Cancer in Asia in the Past Decade- An Update (2000-2012). Asian Pac J Cancer Prev 14: 5567-5577.

32. Boyle P, Ferlay J (2005) Cancer incidence and mortality in Europe, 2004 Annals of Oncology 16: 481-488.

33. Bokor-Bratić M (2003) Prevalence of oral leukoplakia. Med Pregl 56: 552-555.

34. World Health Organization (2015) Prevalence of tobacco smoking. WHO global report on trends in prevalence of tobacco smoking.

35. Allen AM, Oncken C, Hatsukami D (2014) Women and Smoking: The Effect of Gender on the Epidemiology, Health Effects, and Cessation of Smoking. Curr Addict Rep 1: 53-60.

36. Dereje N, Abazinab S, Girma A (2014) Prevalence and Predictors of Cigarette Smoking among Adolescents of Ethiopia. School Based Cross Sectional Survey. Journal of Child \& Adolescent Behavior 3: 2375-4494.

37. White MC, Holman DM, Boehm JE, Peipins LA, Grossman M, et al. (2015) Age and Cancer Risk. Am J Prev Med 46: 7-15.

38. Chandran R (2012) Oral leukoplakia in a South African sample: a clinicopathological study. Oral Dis 19: 592-597.

39. Majumder M, Sikdar N, Paul RR, Roy B (2005) Increased Risk of Ora Leukoplakia and Cancer Among Mixed Tobacco Users Carrying XRCC1 Variant Haplotypes and Cancer Among Smokers Carrying Two Risk Genotypes: One on Each of Two Loci, GSTM3 and XRCC1 (Codon 280). Cancer Epidemiol Biomarkers Prev 14: 2106-2112.

40. Winn DM (2001) Tobacco use and oral disease. J Dent Educ 65: 306-312.

41. World Cancer Research Fund International (2015) Data for cancer frequency by country.

42. Shield KD, Ferlay J, Jemal A, Sankaranarayanan R (2017) The Global Incidence of Lip, Oral Cavity, and Pharyngeal Cancers by Subsite in 2012. CA Cancer J Clin 67: 51-64.

43. Siakholak FR, Ghoncheh M, Pakzad R, Gandomani HS, Ghorat F, et al. (2016) Epidemiology, incidence and mortality of Oral cavity and lips cancer and their relationship with the human development index in the world. Biomed Research and Therapy 3: 872-888.

44. Ferlay J, Soerjomataram I, Dikshit R (2015) Cancer incidence and mortality worldwide: sources, methods and major patterns in GLOBOCAN. Int J Cancer 136: 359-386.

45. Kumar S, Preetha GS (2012) Health Promotion: An Effective Tool for Globa Health. Indian J Community Med 37: 5-12.

46. van der Waal I (2014) US National Library of Medicine.National Institutes of Health. Oral potentially malignant disorders: Is malignant transformation predictable and preventable? 19: 386-390.

47. How Tobacco Smoke Causes Disease: The Biology and Behavioral Basis for Smoking-Attributable Disease. Centers for Disease Control and Prevention (US)(2010).

48. Peterson PE (2003) Tobacco and oral health: the roleof the World health organization. Oral Health Prev Dent 1: 309-315. 